Marolla-Gajardo, J. (2021). Historiadores chilenos frente a la ausencia de las mujeres en la formación del profesorado: los retos desde la didáctica de las ciencias sociales. Revista Electrónica Interuniversitaria de Formación del Profesorado, 24(1), 73-86.

\title{
Historiadores chilenos frente a la ausencia de las mujeres en la formación del profesorado: los retos desde la Didáctica de las Ciencias Sociales
}

Jesús Marolla-Gajardo

Facultad de Educación, Universidad de las Américas, Chile

\section{Resumen}

El estudio trata sobre las concepciones de algunos historiadores chilenos sobre la ausencia y/o presencia de las mujeres y su historia en los cursos de formación del profesorado. Se enfatiza en torno a las posibilidades y las limitaciones que se visualizan al trabajar desde tales perspectivas. El trabajo es un estudio de casos realizado en la ciudad de Santiago de Chile. Se llevó a cabo un análisis de contenido sobre los discursos, y se reflexionó en torno a los marcos teóricos desde la didáctica de las ciencias sociales y el posestructuralismo con perspectiva de género. Los resultados plantean distintas innovaciones y rupturas en torno a la tradicionalidad que existe en la enseñanza de la historia y las ciencias sociales a fin de plantear nuevos espacios de inclusión de la diversidad.

\section{Palabras clave}

Didáctica; inclusión; género; mujeres.

\section{Chilean historians and the absence of women in teacher education: the challenges from the Didactics of Social Sciences}

\section{Abstract}

The study is about the conceptions of some Chilean historians on the absence and / or presence of women and their history in teacher education classes. It emphasizes the

\section{Contacto:}

Jesús Marolla-Gajardo, jesus.marolla@umce.cl Santiago de Chile: Av. José Pedro Alessandri 774, Ñuñoa, CP: 7760197, ORCID: https://orcid.org/0000-0001-6215-0010 
possibilities and limitations that are visualized when working from such perspectives. The methodology is a case study in the city of Santiago de Chile. A content analysis on the discourses was carried out, and the theoretical frameworks were reflected from the social studies education and post-structuralism from a gender perspective. The results raise different innovations and ruptures around the traditionality that exists in the teaching of history and social sciences in order to propose new spaces for the inclusion of diversity.

\section{Key words}

Inclusion; gender; social sciences; women.

\section{Introducción}

El problema del estudio radica en conocer cuáles son las concepciones que manifiestan los participantes sobre la inclusión o la exclusión de las mujeres y su historia en los programas de formación. Es relevante reflexionar sobre el contexto de posibilidades y limitaciones que se entregan desde la historia, a fin de incluir a las mujeres desde perspectivas críticas que problematicen el contenido y planteen opciones que posicionen la justicia social como eje transversal en la formación del profesorado (Crocco, 2018).

Se pueden mencionar trabajos e investigaciones de distintas áreas que han incluido las perspectivas de género en la reflexión educativa. Desde la psicología del aprendizaje y desde el área curricular, estudios como los de Crocco (2018), Azorín (2015), Díaz de Greñu y Anguita (2017) y Woyshner (2002), se han preocupado por la desestabilización de las estructuras patriarcales, y la reflexión en torno al discurso heterosexual y su producción y reproducción en las escuelas. Estudios como los de Pinar (2014), Crocco (2018) y Asián, Cabeza y Rodríguez (2015) se han preocupado por la construcción de los modelos hegemónicos y patriarcales, así como de la segregación de las identidades.

Según estudios como los de Crocco (2018) y Mclaren y Kincheloe (2015), las problemáticas de género obedecen a las estructuras patriarcales, androcéntricas y heteronormativas, bajo las cuales se ha organizado el sistema educativo occidental (Butler, 2006; Foucault, 2008). Esto da cuenta de que muchos de los problemas por razones de género pueden ser compartidos en distintos contextos educativos.

A raíz de lo planteado, los objetivos que se propuestos en el trabajo, se pueden resumir en:

a- Interpretar y comprender el discurso de los historiadores sobre la inclusión de las mujeres y su historia en la formación del profesorado.

b.- Analizar las ventajas y las limitaciones que plantean los participantes para generar cambios ante las desiguales que ocurren en las aulas en torno a la ausencia de las mujeres.

\section{Las concepciones del profesorado y los invisibles en la enseñanza de la historia y las Ciencias Sociales}

Comprendo el concepto de concepciones desde las investigaciones de Ortega (2015), Díez Bedmar (2015), y Marolla (2016). Según los autores, para comprender lo que sucede en las aulas es necesario tener conocimiento de las teorías que guían las acciones del profesorado. Existen principios que orientan sus prácticas y que los llevan a tomar decisiones. 
Ortega (2015), Díez Bedmar (2015) y Marolla (2016). concuerdan en que en las prácticas influyen factores relacionados al conocimiento del contenido, del quehacer pedagógico, del currículo, de los estudiantes, del contexto en que trabaja y sobre todo las finalidades, los propósitos y los valores de la sociedad. Las concepciones del profesorado, por tanto, están en directa relación con el conocimiento base y la ideología que se expresa. Las concepciones se materializan en actividades cotidianas como el proceso de planificación, de enseñanza y de práctica. Marolla (2016) y Ortega (2015) recogen en sus investigaciones que las concepciones del profesorado son un conjunto sistemático de teorías prácticas personales (TPP) que interrelacionan las experiencias personales y sociales con las prácticas.

En general, la presencia de las mujeres es mínima tanto en el currículum, como en los programas y los textos. Azorín (2015), Díaz de Grenu y Anguita (2017) y Pagès y Sant (2012) coinciden en que las narrativas históricas se han enmarcado en el fomento de los valores nacionales, eurocéntricos y masculinos. Se han marginado de los relatos a las mujeres y a los distintos colectivos que representan a la diversidad social. Para los autores anteriores, la historia tradicional (racista, sexista y homofóbica) ha contribuido a la ausencia de las mujeres como de otras personas.

Desde la historiografía de género, algunas autoras y autores (Butler, 2006; Scott, 2008) se han preocupado por rescatar las acciones de las mujeres desde perspectivas como la psicología femenina, el trabajo, las mentalidades y la construcción de espacios corporales y laborales, entre otros temas. Balteiro y Roig-Marín (2015) y Donoso-Vásquez y VelascoMartínez (2013) afirman que la historia no solo se ha construido por los "grandes hombres", sino que el papel de las mujeres, de los niños, las niñas, y de las etnias es fundamental para la comprensión de los procesos y las problemáticas sociales y de género.

\section{La inclusión de las mujeres en la enseñanza de la historia y las Ciencias Sociales}

Autoras como Crocco (2018), Ortega y Pagès (2018), Peinado y García (2015) y Miralles y Gómez (2017) afirman que trabajar con las experiencias de las mujeres podría conllevar las ventajas de alentar a los jóvenes a identificarse más con el pasado y con el presente. Para los autores, sería una forma de luchar contra las desigualdades de género, los prejuicios, los estereotipos y la marginación que han sufrido y que sufren. De esta manera, las alumnas podrían reflexionar sobre quiénes han sido los y las protagonistas de la historia y bajo qué pautas y modelos de género se ha construido la sociedad.

Crocco (2018), Azorín (2015) y Pagès y Sant (2012) dicen que tanto los libros como los programas y el currículum se apoyan fundamentalmente en el protagonismo de los hombres y el de personalidades y masas anónimas. Crocco (2018) y Marolla (2019ay b) coinciden en que los libros y el currículum reflejan en su mayoría a los hombres blancos como protagonistas en temas de economía, política y guerra. Los materiales muestran una historia cargada de estereotipos y de prejuicios en torno a las mujeres, los homosexuales, los indígenas, los inmigrantes, entre otros. Díaz de Greñu y Anguita (2017) y Diez Bedmar (2015) afirman que de esta manera se produce y reproduce el androcentrismo que existe en la sociedad.

Estudios como los de Ortega y Pagès (2018), Peinado y García (2015) y Miralles y Gómez (2017) coinciden en que la construcción de los textos y el currículum es realizada desde las estructuras androcéntricas de la sociedad. Las mujeres son presentadas desde espacios privados y domésticos, transmitiendo roles subordinados sobre sus acciones e historia. Pagès y Sant (2012, p. 102) coinciden y agregan que los libros son construidos desde cinco 
perspectivas diferentes: 1) la historia sin mujeres; 2) la historia y las mujeres; 3) la historia de las mujeres; 4) la historia para las mujeres y; 5) la historia desde las mujeres.

Las investigaciones de Marolla (2019a), Fernández (2015) y Gómez y López (2014) coinciden en que en los libros de textos las mujeres son visibilizadas solo en algunas secciones. La imagen que se proyecta sobre ellas está construida desde visiones androcéntricas y cargadas de prejuicios y estereotipos. Ortega y Pagès (2018), Peinado y García (2015) y Crocco (2018) afirman que para plantear la enseñanza desde la perspectiva de género con la inclusión de las mujeres, será necesario reinterpretar la historia y establecer un espacio en donde se deje en evidencia el rol que han cumplido tanto las mujeres, como el resto de personas que han sido invisibilizadas en la sociedad y en las narrativas historiográficas.

\section{La formación del profesorado y la inclusión de las mujeres y su historia}

Distintos y distintas autoras han reflexionado sobre la formación y las prácticas que ha realizado el profesorado desde la inclusión de las mujeres como personas y protagonistas de la historia (Crocco, 2018; Azorín, 2015; Woyshner, 2002). Las conclusiones a que han llegado es que los trabajos sobre la formación y la inclusión de las perspectivas de género son escasos. Los pocos que existen tratan sobre el género, la diversidad, la igualdad, la discriminación, entre otros temas, de forma aislada dentro del campo educativo.

Ortega y Pagès (2018) y Peinado y Luque (2015) a través de sus investigaciones, afirman que el profesorado actúa como un agente en la transmisión de los estereotipos, de los prejuicios y de las desigualdades de género. La formación, por tanto, está basada en las estructuras androcéntricas, los estereotipos sexuales y la universalización de los valores masculinos (Rodríguez, 2014).

Crocco (2018) y Woyshner (2002) coinciden en que una manera de abordar los problemas por razones de género, es que se incluyan a las mujeres en la formación y en las aulas, desde la problematización de los estereotipos, los prejuicios y las desigualdades que existen y que se reproducen en la sociedad. Las autoras afirman que la ausencia de las mujeres es debido a que el profesorado tiende a enseñar desde el modelo "más valorado socialmente", o sea, desde lo masculino. Implícitamente se transmiten jerarquías sexuales y de género que reafirman y reproducen las estructuras sociales de desigualdad.

Crocco (2018), Balteiro y Roig-Marín (2015) y Donoso-Vásquez y Velasco-Martínez afirman que si se incluyera a las mujeres se podría incentivar a los y a las estudiantes a que se empoderen y se comprometan con las transformaciones de las problemáticas de género. Crocco (2018) plantea que para trabajar con las mujeres en la historia debe existir un cambio de enfoques en las prácticas realizadas. Marolla (2019b), Azorín (2015), Díez de Greñu y Anguita (2017) y Ortega y Pagès, agregan que las prácticas del profesorado deben favorecer e impulsar un discurso que potencie la inclusión, la empatía y los espacios de agencia y empoderamiento.

Los autores anteriores han concluido que el profesorado debe cambiar los enfoques tanto en la formación como en las prácticas a fin de incluir a las mujeres como personajes y protagonistas de la historia enseñada. Para luchar contra los estereotipos y los prejuicios, hay que plantear la inclusión desde la problematización y la deconstrucción de las estructuras de género. Las mujeres y su historia no se deberían incluir como un apéndice, sino que la inclusión debería ser transversal. 


\section{La perspectiva de género como fundamento para investigar}

En la investigación se consideran los planteamientos de los estudios de género desde las corrientes posestructuralistas (Apple, 2014; Mclaren \& Kincheloe, 2015). Desde estas perspectivas se elabora un discurso de crítica y de posibilidad a fin de que los grupos subordinados y excluidos comprendan sus mundos e historias sociales.

En torno a las identidades, desde la teoría queer se han cuestionado tales construcciones como espacios únicos y cerrados sobre sí mismos (Butler, 2006). Se propone la subversión de las categorías de sexo y de género desde la naturalización de las identidades (Solana, 2013). Brah (2011) afirma que las identidades no son fijas, sino que responden a una multiplicidad de relaciones que están en constante movimiento. Las categorías de identidad, como de género, son expresiones construidas en base a la diferencia sexual más que constructos que obedecen a procesos naturales (Scott, 2008).

Las identidades, siguiendo a Butler (2006) y Foucault (2008) se construyen dentro de complejas interacciones entre el discurso y el poder, y éste se entiende en función de las convenciones culturales heterosexuales. Butler (2006) dice que el género no se ha construido de forma coherente, sino que depende del contexto, de la raza, de la clase, de la etnia y de la sexualidad. Factores que, generalmente, están determinados por las convenciones heterosexuales. Butler (2006) coincide con Foucault (2008) al reconocer que el género es una construcción social formada por el poder y el saber, y agrega que la crítica debe visibilizar cómo operan, cómo se construyen y cómo se instauran tales estructuras.

Scott (2008) coincidentemente, dice que se ha escrito excluyendo a las mujeres y a los estudios de género, debido a que la cultura ha sido patriarcal. Agrega que la masculinidad configura sus símbolos y sus prácticas, las cuales excluyen las acciones de las mujeres. Si se les incluye, equivaldría a reconocer que ellas en sus subjetividades, están en los mismos niveles que el patriarcado. Butler (2006), Scott (2008) y Azorín (2015) coinciden en que desde la construcción de género, los términos "masculino" " "femenino" son intercambiables; cada uno tiene una historia social, la cual varía en función de las restricciones socioculturales.

Aguilar (2015), Ortega y Pagès (2018) y Díaz de Greñu y Anguita (2017) y afirman que se debe repensar y subvertir la construcción patriarcal de la escuela, con el objetivo de crear espacios de igualdad y de diferencia, haciendo visible las múltiples identidades y sexualidades. Para superar dificultades como la violencia, la discriminación, la subordinación, entre otras problemáticas, se debe trabajar para comprender, reflexionar y reconstruir las categorías de identidad y de género desde la diversidad y la democracia.

\section{Metodología}

El paradigma de la investigación es cualitativo (Álvarez-Gayou, 2003; Fernández y Johnson, 2015), con foco en las expresiones y los problemas de los grupos que han sido silenciados. El objeto de estudio se centra en la explotación, la opresión y la falta de autonomía de las mujeres (Cohen et al., 2007).

La investigación tiene el enfoque del estudio de casos (Ortega, 2015; Marolla, 2016; Simons, 2011). Este enfoque es utilizado cuando el interés del estudio está centrado en un fenómeno, una población o una condición general. El enfoque es un estudio de caso exploratorio (Yin, 2015) e interpretativo.

Entre algunas características de los estudios de caso (Álvarez-Gayou, 2003), es posible comentar que se trabaja con datos no estructurados, el análisis es de tipo cualitativo y el objetivo es comprender los propios casos y no generalizar a toda la población. El diseño está 
compuesto por la conjunción de los planteamientos de Wolcott (1994). Wolcott (1994) plantea tres fases: descripción, análisis e interpretación. Creswell (2014) a su vez entrega cuatro fases: descripción (¿qué sucede?), información (¿qué significa?), confrontación (¿por qué sucede?), y reconstrucción (¿cómo podría ser diferente?).

\section{Procedimiento y diseño de investigación}

Para la investigación, el diseño de análisis cualitativo está construido por las fases de análisis de Creswell (2014). La organización de códigos, la reducción, la exposición y la verificación de los datos con los planteamientos de Simons (2011) y Fernández y Johnson (2015). La metodología de Wolcott (1994) y Simons (2011) para la transformación de los datos, y los planteamientos de Goldstein (2017) y Finlay (2015) para la reflexión deconstructiva de los discursos. Las fases seguidas en el estudio, de manera consecutiva, se pueden resumir en:

1) Fase de reducción y selección de información (Creswell, 2014).

2) Fase analítica. Se categorizan los datos en función de las preguntas y los objetivos (Creswell, 2014; Simons, 2011; Fernández y Johnson, 2015).

3) Fase interpretativa de casos. Se realiza el análisis de contenido (Bardin, 1986), se organizan los datos en patrones, conceptos, esquemas y, además, se produce la triangulación de los datos (Creswell, 2014; Simons, 2011).

4) Fase ideológica interpretativa. Se plantea desde el análisis de contenido (Bardin, 1986). Se realizan dos procesos:

5) Etapa discursiva para la emancipación (Goldstein, 2017; Wolcott, 1994).

6) Deconstrucción: se genera un planteamiento para la emancipación desde el género y la didáctica de las ciencias sociales, desde la teoría crítica (Goldstein, 2017), la teoría feminista (Finlay, 2015) y la justicia social (Finlay, 2015; Goldstein, 2017).

Los participantes se componen de 4 historiadores que pertenecen a distintas universidades de Santiago de Chile. Los criterios utilizados para la elección, siguiendo a Creswell (2014) y Simons (2011) fueron: 1) La facilidad para acceder y permanecer en el campo; 2 ) la existencia de diversos procesos, programas, interacciones y personas; 3 ) la posibilidad de establecer una buena relación con los informantes; 4) la posibilidad de asegurar la calidad y credibilidad del estudio; 5) la ubicación geográfica y; 6) la disposición de las instituciones y de las personas que vayan a participar.

Además, siguiendo a Simons (2011), los participantes escogidos no cumplen la finalidad de representatividad, ya que dentro de los objetivos de los estudios de caso no se pretende la generalización. En la tabla 1 se muestran quienes participaron en el estudio:

Tabla 1.

Historiadores participantes

\begin{tabular}{cccc}
\hline Nombre & Tipo de Universidad & Experiencia laboral & Perspectiva histórica \\
\hline Nicole & Pública & Más de 30 años. & $\begin{array}{c}\text { Historia de género y } \\
\text { psicohistoria. }\end{array}$ \\
\hline Carla & Privada & Más de 20 años. & Historia de género y social. \\
\hline Jaime & Pública & Más de 30 años. & Historia social y política. \\
\hline Simón & Privada-Cristiana & Más de 30 años. & Historia cultural y política. \\
\hline
\end{tabular}


Los resultados son producto de entrevistas semi-estructuradas (Simons, 2011). La estructura se ha compuesto, siguiendo a Álvarez-Gayou (2003), Creswell (2014) y Simons (2011), en entrevistas como conversaciones. Se busca generar un clima y una relación que posibilite que los informantes puedan compartir sus experiencias y opiniones con libertad. Este tipo de entrevista destaca por ser proactiva y persigue que se reflexione sobre las prácticas y los discursos.

Las entrevistas fueron grabadas. Nos basamos en los criterios de confidencialidad y anonimato (Simons, 2011; Cohen et al., 2007). asegurando a los y las participantes el resguardo ante cualquier información sensible y personal. Tanto para las personas como las instituciones se utilizaron seudónimos ${ }^{1}$. Las principales preguntas realizadas en las entrevistas se señalan en la tabla 2. Cabe destacar que desde tales preguntas emergen los temas que se plantean en los resultados.

Tabla 2.

Preguntas de las entrevistas

\section{Preguntas de la entrevista}

¿Cuáles son las finalidades de la enseñanza de la historia?

¿Cuáles son los principales temas que se investigan en la historia?

¿Cómo los tema que se investigan en la historia influyen en los programas de formación del profesorado?

¿Cuáles son las ventajas y los inconvenientes para incluir a las mujeres y su historia en los programas de formación?

¿Qué cambios se deberían realizar para incluir a las mujeres y su historia en los programas de formación?

\section{Las mujeres y su historia en la formación del profesorado}

En general, los historiadores dicen que las experiencias de las mujeres en la formación no son invisibles ni están ausentes. No obstante, Jaime, Nicole y Carla coinciden en que el androcentrismo y el patriarcado que existe en la sociedad han impedido que se planteen perspectivas que problematicen los discursos que se entregan sobre la participación de las mujeres en la historia.

Jaime reconoce que la historia social tiene pendiente la inclusión crítica de las mujeres. Para él: "... es un tema de poder, como todas esas cosas... el patriarcalismo es un rasgo muy enquistado en nuestra cultura y en nuestras relaciones sociales y de muy larga duración..." (Jaime, 11 de diciembre de 2014). Aún se sigue dando prioridad a la historia tradicional y de los grandes hombres. La inclusión, para el historiador, implica transformar las estructuras sociales y reformular el cómo se entiende la historia.

\footnotetext{
${ }^{1}$ Se ha optado por la presentación de nombres y no códigos para la identificación de los participantes a fin de identificar de forma clara cada discurso.
} 
Nicole y Carla comentan que las escuelas más que generar transformaciones, han colaborado en consolidar relaciones y prácticas discriminatorias y patriarcales. Nicole dice que: “... la educación tiende a reproducir los patrones consabidos más que adentrarse en lo nuevo. Yo diría que en ese contexto, la educación más que abrir puertas, lo que ha hecho tradicionalmente es cerrar puertas... lo que ha hecho persistentemente es tomar prevenciones respecto de un cambio exagerado..." (Nicole, 23 de Diciembre de 2014).

Para Nicole, los historiadores son los responsables de forma indirecta de la construcción historiográfica que solo ha destacado a los hombres. Para ella, las corrientes de género no solo buscan visibilizar a las mujeres en igualdad a los hombres, sino que plantean que se deben deconstruir las perspectivas tradicionales en que se ha construido el conocimiento:

Toda la invisibilidad de las mujeres ha sido una suerte de construcción... Porque las mujeres no es que hayan sido invisibles, esa es una construcción, una distorsión de la realidad... Hay una suerte de política activa de discriminación que siempre va a estar... de manera que este protagonismo sigue siendo invisible (sic). (Nicole, 23 de diciembre de 2014).

Carla coincide con Nicole en torno al dominio patriarcal y hegemónico que existe en los cursos de historia. Es necesario plantear nuevas metodologías y nuevas temáticas para la enseñanza y el aprendizaje. Ella dice que los programas de su carrera se han construido en función de los problemas de raza, clase y género: “... no significa que van a ser expertos en historia de muchas mujeres, pero sí en el momento de situarse en una sala de clases van a tener otras perspectivas" (Carla, 24 de noviembre de 2014).

Según Carla, se deben considerar aspectos como la clase, la etnia y el género desde perspectivas problemáticas. Hay que generar rupturas con las lógicas tradicionales de la enseñanza, como así también reconocer que las problemáticas provocadas por la invisibilidad de las mujeres no son producidas solamente por razones de género. Los problemas de género deben ser abordados desde la multidisciplinariedad y la transversalidad en la formación: "Es un problema de cómo nosotros abordamos el conocimiento, de cómo abordamos la historia, de cómo abordamos las perspectivas analíticas, es transversal..." (Carla, 24 de noviembre de 2014).

\section{Las ventajas y las limitaciones de la inclusión de las mujeres en la formación del profesorado}

Los historiadores plantean que la historia debería servir para 1) conocer el presente y proyectarse hacia el futuro y; 2) analizar los problemas de la sociedad para empoderarse como ciudadanos y ciudadanas. Para Jaime, la historia debería posibilitar la construcción de una ciudadanía empoderada que actúe para la construcción de un mejor futuro:

Para que cualquier ser humano se ubique en el mundo en que le toca vivir y sepa para dónde quiere ir... para sensibilizar a las personas frente a lo que son nuestras carencias, nuestras necesidades más urgentes y ver cuál es el origen de esas carencias. (Jaime, 11 de diciembre de 2014).

Para Carla es fundamental que la historia tenga una finalidad de formación ciudadana. Ella comprende la historia como un saber subjetivo que plantea conflictos políticos e ideológicos sobre cómo se entiende y cuáles son los compromisos que se asumen en la sociedad: 
Yo diría que para mí la historia es un ámbito de construcción de ciudadanía ... La historia para mí son posiciones políticas. Tiene que ver con situarse como sujetos... Yo como mujer, como mujer con un determinado pensamiento político, un determinado pensamiento frente a la vida... (Carla, 24 de noviembre de 2014).

Carla y Nicole enfatizan que a través del estudio de la historia se debe reflexionar sobre cómo se ha construido el conocimiento y cómo se han construido las identidades de las personas. Nicole afirma que: “... la historia sirve básicamente para dar conciencia de sujeto de su propia identidad ... de su pertenencia a un aquí y a un ahora ..." (Nicole, 23 de diciembre de 2014). De esa manera se puede dar reconocimiento a la diversidad de identidades que existe en la sociedad.

Los participantes afirman que existen múltiples posibilidades para incluir a las mujeres en la enseñanza de la historia y en la formación. Simón dice que el tratamiento de los problemas por razones de género en distintos cursos, contribuiría a fomentar el respeto por la diversidad y la tolerancia hacia las diferencias. Agrega que la formación debería plantearse desde la diversidad de temas, problemas y personajes a fin de que se reflexione ante la realidad actual.

Según Jaime, en su programa se entregan distintas herramientas para incluir y problematizar las experiencias de las mujeres. No obstante, reconoce que no existen especialistas en los estudios de género, ni se fomentan tales perspectivas. Nicole y Carla, por su parte, coinciden en que la inclusión debe fomentar la transformación y no la reproducción de las estructuras que han oprimido a las mujeres. Nicole dice que se debe trabajar para transformar las prácticas tradicionales que se realizan en las escuelas, donde se producen y reproducen los roles, los estereotipos, los prejuicios y las jerarquías basadas en el género.

Nicole agrega que es fundamental reflexionar sobre el trabajo pedagógico, ya que ahí es donde se socializa, se transmite y se normalizan los roles y los comportamientos de las personas:

Porque la educación lo que hace es adentrar al sujeto en un entorno de socialización, en el contexto en el cual él aprende los hábitos, aprende las normas, aprende el comportamiento, aprende las relaciones sociales. Desde que son chicos no se les enseña que el rol de la mamá es tan relevante como el del papá, de manera que las mujeres aparecen muy pasivas, muy subordinadas, muy enquistadas en acciones que no cambian. En cambio, los hombres son los aventureros, son los agresivos, son los exploradores del espacio... (Nicole, 23 de diciembre de 2014).

Nicole propone que la escuela trabaje desde lógicas que consideren las perspectivas de género de la diferencia. La historiadora reconoce que es una temática compleja, ya que ni la escuela ni la sociedad se ha articulado para reconocer tales perspectivas. La inclusión de las mujeres, agrega, no pasa por su trabajo en las aulas o la formación, sino que conlleva esfuerzos por realizar cambios sociales: “... si la sociedad se lee en clave masculina, difícilmente un historiador va a mirarla en clave femenina...". (Nicole, 23 de diciembre de 2014).

La inclusión, por tanto, debe enfocarse no en aportar mayores conocimientos, sino que en la finalidad del cambio social y la transformación de las estructuras que han generado fuertes estereotipos, prejuicios y desigualdades entre las personas. Carla y Nicole, coinciden, en que tales disposiciones han sido creadas por los hombres para oprimir a las mujeres. 
Carla afirma que aún existen fuertes estructuras sociales que discriminan, y que son producidas por un patriarcado específico que ha monopolizado el poder económico, político e intelectual: “... aquí me estoy refiriendo no solamente a hombres, sino que es mucho más restringido. Son hombres, son blancos, tienen poder económico, son "racionales"”. (Carla, 24 de noviembre de 2014). Afirma que las ventajas de la inclusión radican en que las escuelas podrían trabajar para la transformación de las estructuras opresivas que ha creado el patriarcalismo.

La profesora dice que, aunque se ha dado presencia y se ha fomentado la participación de las mujeres en distintas áreas de la sociedad, en la práctica sus acciones siguen bajo evaluación y, por tanto, subordinación masculina. Aún existen muchas violencias cotidianas que es necesario erradicar. Se debe considerar la inclusión de las mujeres y su historia con un objetivo de igualdad social: “... yo creo que la historia y la educación son centrales.” (Carla, 24 de noviembre de 2014). Para la historiadora es necesario repensar la historia y su enseñanza: "Yo creo que aún es una formación que le debe mucho. Sin embargo, me parece que cada vez hay más sensibilidades, hay más interés por participar." (Carla, 24 de noviembre de 2014).

Por último, ella reconoce que el trabajo en las aulas es fundamental. En estos espacios se pueden propiciar marcos que posibiliten los cambios o, al contrario, se puede contribuir a la reproducción y a la perpetuación de las estructuras desiguales y opresivas de la sociedad. En la actualidad, la formación se ha construido en base a las estructuras patriarcales, de poder, y desde la discriminación por razones de género. Para ella, aquellas son las estructuras que se deben repensar y transformar, ya que en los programas actuales:

... siguen reproduciendo viejos patrones sociales en que las mujeres aparecen más relegadas, produciéndose entonces estas clásicas tensiones que hoy día vemos, entre mujeres con mucha figuración en los ámbitos de la economía, pero no necesariamente relevadas desde un punto de vista de social y menos, reconocida su actuación en la educación... (Carla, 24 de noviembre de 2014).

\section{Discusión y conclusiones: las ventajas y los obstáculos para la inclusión en la formación del profesorado}

Los historiadores han coincidido en que las escuelas transmiten normas, comportamientos y patrones sociales. Desde el paradigma crítico, Mclaren \& Kincheloe, (2015) y Apple (2014) plantean que la transmisión de las estructuras sociales de poder, privilegia unos contenidos y a unos protagonistas por sobre otros. La escuela y la enseñanza han mantenido el status quo que han designado las elites que se encuentran en el poder. Pagès y Sant (2012), Azorín (2015) y Apple (2014) coinciden en que se ha marginado a las mujeres y su historia, privilegiando el tratamiento de los valores nacionales, eurocéntricos y masculinos.

Los participantes coinciden en que la enseñanza de la historia y las ciencias sociales, más que cambiar las estructuras hegemónicas de género, las reproduce. La escuela, por tanto, colabora en esta tarea socializando y normalizando los comportamientos, las identidades y las distintas expresiones de género. Foucault (2008) dice que la tradición podría ser el constructo bajo el cual se han validado y se han normalizado las estructuras sociales y las jerarquías que han generado las opresiones y las subyugaciones sociales.

Scott (2008), Balteiro y Roig-Marín (2015) coinciden con los comentarios de los participantes en que la historia se ha construido en base a una jerarquía que ha posicionado el rol de los 
hombres como protagonistas en una historia que, además, los ha destacado como soldados, científicos y líderes por sobre lo que se trabaja de las mujeres. El rol que se presenta sobre las mujeres está ligado a planos domésticos y privados (Díaz de Greñu y Anguita, 2017).

Pagès y Sant (2012) y Apple (2014) y Mclaren \& Kincheloe, (2015) coinciden con los participantes en que las estructuras de la formación del profesorado son definidas de forma intencionada por quienes monopolizan el poder. Tal poder está basado en las estructuras patriarcales y la reproducción de los estereotipos, los prejuicios y las desigualdades de género. Para Scott (2008), Díez Bedmar (2015), Fernández (2015) y Butler (2006) la hegemonía patriarcal de la sociedad se ha encargado de normalizar las diferencias y los roles de las mujeres, posicionando en el poder a los hombres.

Crocco (2018) y Azorín (2015) coinciden con lo planteado por los participantes, donde la reproducción de las desigualdades de género se transmite a través a de la construcción del currículo, la formación profesional y las prácticas que se realizan. Afirman que el currículo se ha formulado desde una cronología creada por los hombres para validar y destacar sus acciones. Por tanto, en la formación del profesorado se construye una narrativa cronológica en la que los protagonistas son hombres que destacan en temas políticos, económicos y militares (Marolla, 2019a y b; Díez Bedmar, 2015; Fernández, 2015; Pagès y Sant, 2012).

Butler (2006), Aguilar (2015), Azorín (2015) y Díaz de Greñu y Anguita (2017) plantean, coincidiendo con los participantes, que la división entre los roles de género que se transmiten y reproducen, no se presenta como una construcción, al contrario, se presentan de manera naturalizada. Esto provoca que el discurso que se transmite no incentive la participación de las mujeres, ya que está basado en estructuras desiguales por razones de género. Foucault (2008) Ilama a esto "estructuras de verdad" en la historia. Son construcciones culturales y de conocimiento que actúan como códigos y discursos que se transmiten en la sociedad. El poder está definido de acuerdo a quienes controlan, difunden y producen tales estructuras.

Según Apple (2014), Mclaren \& Kincheloe (2015), Ortega y Pagès (2018) y Rodríguez Martínez (2014), los roles y las narrativas desiguales sobre las mujeres, provoca que se las visibilice desde discursos que impiden que las chicas y los chicos encuentren modelos de mujeres activas y empoderadas. Esto podría tener el efecto de que los y las estudiantes no se identifiquen con los personajes de la historia, y de esa manera no participen en la sociedad.

Marolla (2019a), Ortega y Pagès (2018), Crocco (2018) y Fernández (2015) coinciden en que se ha presentado una visión de las mujeres desde perspectivas subalternas. Esto provoca que se construya una imagen sobre ellas como "espectadoras" de la historia, en donde los protagonistas han sido los hombres. Las mujeres han tenido una participación parcelada, anecdótica y han sido tratadas como un objeto corporalizado y sexualizado (Crocco, 2018; Ortega y Pagès, 2018; Scott, 2008).

De lo anterior, es posible concluir que en la formación del profesorado se han "negado" las expresiones de diversidad de género. Se han negado las posibilidades de problematizar las construcciones que han posicionado a los hombres por sobre las mujeres (Scott, 2008). Las mujeres han sido presentadas desde la "diferencia" y la "exclusión" (Fernández, 2015; Díez Bedmar, 2015; Díaz de Greñu y Anguita, 2017 y Azorín, 2015). Los roles y los discursos se han presentado desde un "otro" subordinado y "naturalizado" a las acciones que realizan los hombres con poder.

Para algunos autores y autoras (Crocco, 2018; Ortega y Pagès, 2018; Díaz de Greñu y Anguita, 2017, Díez Bedmar, 2015; Woyshner, 2003), coincidiendo con los participantes, el problema no es la inclusión de las mujeres, sino que es desde dónde y qué están transmitiendo y 
produciendo los discursos y las prácticas que trabajan con su presencia, sus problemas y sus roles en la formación, en las aulas y en la sociedad.

Por lo anterior, en cuanto a ventajas, con el fin de favorecer el empoderamiento ciudadano y la participación activa de las chicas, se debería trabajar desde estructuras que fomenten la problematización con el objetivo de construir identidades de género alternativas a las tradicionales. Ortega y Pagès (2018), Crocco (2018), Azorín (2015) y Marolla (2019b) coinciden en plantear que para la construcción de modelos de identidad alternativos se debe exponer la normalización, la subordinación, los prejuicios y los estereotipos que han girado en torno a las mujeres.

A través de la empatía histórica (Díez Bedmar, 2015 y Díaz de Greñu y Anguita, 2017) y el cuestionamiento de los discursos sobre la identidad y los roles que se reproducen y transmiten en la formación, se podrían generar modelos que favorezcan el empoderamiento crítico y la lucha por las desigualdades de género. Las narrativas históricas deben reconstruirse considerando las experiencias de vida, las subordinaciones y las posibilidades de emancipación para las mujeres en todos los espacios sociales y las etapas históricas (Woyshner, 2002).

Butler (2006), en este sentido, afirma que no existen identidades predeterminadas, sino que son expresiones performativas del género. El trabajar los roles y las identidades de género desde modelos alternativos a los tradicionales, debería promover que se fomentara el empoderamiento ciudadano, la participación y lucha en contra las desigualdades por razones de género. Una propuestos podría ser la subversión de las estructuras y de los discursos que han naturalizado la subordinación y las desigualdades (Miralles y Gómez, 2017; Marolla, 2019a y b; Díaz de Greñu y Anguita, 2017; Azorín, 2015). Se debe repensar la historia y las narrativas a fin de que se presenten a las mujeres como protagonistas en una historia que las ha excluido (Croccro, 2018; Aguilar, 2015; Ortega y Pagès, 2015; Díez Bedmar, 2015).

\section{Referencias}

Aguilar Ródenas, C. (2015). Igualdad, género y diversidad sexual en la Formación Inicial de Maestro/a en la Universidad Jaume I (UJI). Temas de educación, 21(1), 77-96.

Álvarez-Gayou, J. L. (2003). Cómo hacer investigación cualitativa. Fundamentos y metodología. México: Paidós.

Apple, M. W. (2014). Official knowledge. Democratic education in a conservative age. New York: Routledge.

Azorín, C. Ma (2015). Análisis de la política de género en España: hacia una educación inclusiva y coeducadora. En A. B. Mirete y R. Nortes (Eds.), Investigación e Innovación: una constante necesaria en la formación del profesorado (pp. 1-12). Murcia: Universidad de Murcia.

Bardin, L. (1986). El análisis de contenido. Madrid: AKAL.

Brah, A. (2011). Cartografías de la diáspora. Identidades en cuestión. Madrid: Traficantes de sueños.

Britzman, D. (2002). La pedagogía transgesora y sus extrañas técnicas. En R. Mérida, (Ed.), Sexualidades transgresoras. Una antología de estudios queer (pp. 197-228). Barcelona: Icaria.

Butler, J. (2006). Deshacer el género. Barcelona: Paidós. 
Cohen, L.; Manion, L. y Morrison, K. (2007). Research Methods in Education. London: Routledge.

Creswell, J. (2014). Research design. Qualitative, Quantitative and Mixed Methods Approaches. California: Sage.

Crocco, M. S. (2018). Gender and sexuality in history education. En The Wiley international handbook of history teaching and learning (pp. 335-364). Hoboken, NJ: Wiley.

Díaz de Greñu, S. y Anguita, R. (2017). Estereotipos del profesorado en torno al género y a la orientación sexual. Revista Electrónica Interuniversitaria de Formación del Profesorado, 20(1), 219-232.

Donoso-Vázquez, T. y Velasco-Martínez, A. (2013). ¿Por qué una propuesta de formación en perspectiva de género en el ámbito universitario? Profesorado. Revista del Currículum y Formación del Profesorado, 17(1), 71-88.

Fernández, A. (2015). Género y enseñanza de la Historia. En $M^{a} A$. Domíngez Arranz y R. $M^{a}$ Marina (Eds.), Género y enseñanza de la Historia: silencios y ausencias en la construcción del pasado (pp. 29-55). Madrid: Sílex.

Fernández, M. B. y Johnson M., D. (2015). Investigación-acción en formación de profesores: Desarrollo histórico, supuestos epistemológicos y diversidad metodológica. Psicoperspectivas, 14(3), 93-105.

Finlay, L. (2015). Probing Between: Reflexive-relational approaches to human science research. En, C. Fischer, L. Laubscher, \& R. Brooke (Eds.). Invitation to Psychology as a Human Science (pp. 55-84). Pittsburgh, PA: Duquesne University Press.

Foucault, M. (2008). Historia de la sexualidad 1: la voluntad del saber. Buenos Aires: Siglo XXI.

Goldstein, S. E. (2017). Reflexivity in narrative research: Accessing meaning through the participant-researcher relationship. Qualitative Psychology, 4(2), 149164. https://doi.org/10.1037/qupoo00035

Gómez Carrasco, C. J. y López Martínez, A. Ma (2014). Las imágenes de los libros de texto y su función en la enseñanza de la historia. Diseño de un instrumento de análisis. Enseñanza de las Ciencias Sociales: Revista de Investigación, 13, 17-29.

Macbeth, D. (2001). Reflexivity in Qualitative Research: Two Readings, and a Third. Qualitatitve Inquiry, 7(35), 35-68.

Marolla, J. (2016). La inclusión de las mujeres en enseñanza de la historia y las ciencias sociales. didáctica de las ciencias sociales. Estudio colectivos de casos en las aulas chilenas sobre sus posibilidades y limitaciones. (Tesis Doctoral inédita). Universidad Autónoma de Barcelona, España.

Marolla, J. (2019a). La Didáctica de las Ciencias Sociales y el problema de la ausencia de las mujeres y su Historia. Reflexiones en torno a un estudio de casos para transformar las prácticas de enseñanza. Revista Electrónica Educare, 23(1).

Marolla, J. (2019b). La inclusión de las mujeres en las clases de historia: posibilidades y limitaciones desde las concepciones de los estudiantes chilenos. Revista Colombiana de Educación, 78, 1-24.

Mclaren, P. \& Kincheloe, J. L. (2015). Pedagogía crítica. De qué hablamos, dónde estamos. Barcelona: Graó. 
Miralles, P. y Gómez, C. (2017). Enseñanza de la historia, análisis de libros de texto y construcción de identidades colectivas. Historia y Memoria de la Educación, 6, 9-28.

Ortega, D. (2015). Didáctica de la Historia y construcción de la identidad cultural iberoamericana en el currículo y libros de texto de Ciencias Sociales de Educación Primaria españoles. (Tesis Doctoral inédita). Universidad de Burgos, Burgos.

Ortega, D. y Pagès, J. (2018). Género y formación del profesorado: análisis de las Guías Docentes del área de Didáctica de las Ciencias Sociales. Contextos Educativos. Revista de Educación, 21, 53-66.

Pagès, J. y Sant, E. (2012). Las mujeres en la enseñanza de la Historia: ¿Hasta cuándo serán invisibles? Cad. Pesq. CDhis, 25(1), 91-117.

Pinar, W. (2014). La teoría del currículum. Madrid: Narcea, ediciones.

Rodríguez Martínez, C. (2014). La invisibilidad de las mujeres en los contenidos escolares. Cuadernos de Pedagogía, 447, 32-35.

Scott, J. (2008). Género e Historia. México: FCE.

Simons, H. (2011). El estudio de caso: Teoría y práctica. Madrid: Morata.

Solana, M. (2013). La teoría queer y las narrativas progresistas de identidad. Revista de estudios de género, La Ventana, IV(37), 7-105.

Spivak, G. (1992). French Feminism Revisited: Ethics and Politics. En, J. Butler y J. Scott (Eds), Feminist Theorize the Political (pp. 54-85). New York: Routledge.

Wolcott, H. (1994). Transforming Qualitative Data: Description, Analysis and Interpretation. London: Sage.

Woyshner, C. (2002). Political History as Women's History: Toward a More Inclusive Curriculum. Theory and Research in Social Education, 30(3), 354-380.

Yin, R. (2015). Case Study Research: Design and Methods. Thousand Oaks, CA: Sage. 